International Journal of Research in Advent Technology, Vol.7, No.3, March 2019

E-ISSN: 2321-9637

Available online at www.ijrat.org

\title{
Iot Based Officers Tracking System
}

\author{
${ }^{1}$ Mr.M.Annamalai, ${ }^{2}$ Mr.B.Sundaramurthy, ${ }^{3}$ G.Divya \\ ${ }^{1}$ Assistant Professor, ${ }^{2}$ Associate Professor, ${ }^{3}$ Final year MCA \\ Department of Computer Science \& Engineering Vinayaka Mission's Kirupananda Variyar Engineering College, Vinayaka \\ Mission's Research Foundation (Deemed To Be University) Salem - 636 308,TamilNadu, India. \\ annamalaim@vmkvec.edu.in, sundaramurthy@vmkvec.edu.in,divyawelcomeyou@gmail.com
}

\begin{abstract}
Currently, industries, organizations are using personal identification ways like RFID, Iris recognition, for Fingerprint identification for monitoring employees. Amongthese personal identification ways as well as face recognition is most natural, less time is taken and high economical one despite being troublesome to implement, many applications attending management systems and security systems. In this work, a system is enforced that tracks employees throughout office also in industries and etc. using face detection and recognition technology. A period is about for taking attending and also the info is mechanically uploaded into the online server through the web property. This process is done without any human intervention. In the system a Raspberry Pi put in with OpenCV library and a Raspberry Pi Camera module is connected for facial detection and Recognition. The data is kept within the memory card connected to Raspberry Pi and it will be accessed through the web. The results show thatcontinuous observation increases accuracy and maximizes the output.
\end{abstract}

Keywords - Internet of Things (Iot), Raspberry pi,Face recognition.

\section{INTRODUCTION}

The Currentmethodsthat used for tracking availability of officers in their office is through manual attendance system and also throughRFID primarily based, BIO-METRIC primarily based AND MATLAB primarily based. Hence, it's vital to build some application to monitor the officers periodically through some automation methods. Anotheradvantage of those sorts isthat we can able to reduce work of manpower and human error. In order to achieve this some open commandvisualization (OPEN-CV) is used through Raspberry pi which is used for most of the web based Iot application. It is an open source libraryin which the source code is openand it is useful in visual field like Image processing. The mainaimof this open platform is to require and manage group action victimization using FACE RECOGNITION.

\section{LITERATURE REVIEW}

Many organizations, companies and institutions are taking periodic attendance using [1] RFID methods, [2] Biometric Fingerprint method and Registers. These methods generally take more time for calculation. Also, major issue with this RFID (Radio Frequency Identification) [1] is that it uses electromagnetic fields to automatically identify and track tags attached to persons. RF signals can breach the privacy and security of human beings. RFID strategies ultimately effect software that allows each person to be analyzed by the primary database. This environment can be easily affected by hackers. If input from the userend is not proper then it leads to less read rate therefore many problems will occur with person identification.
The fingerprint identification method using biometric systems employ fingerprint as a unique identity One of the most accurate systems running effectively today is biometric system. The recognition of separate persons fingerprint from a set of enrolled fingerprints is a little tedious process. At the same time, it may not give any information about the original fingerprint. This theory already has been stated false as many algorithms [3] reveal that a fingerprint can be reconstructed with minute templates.

Iris Recognition [4] is another type of implementation where the iris of people is scanned, stored and then retrieved for the comparison and attendance is managed automatically in the server. But we may have some difficulty in capturing iris of the students or employees and hence a fast implementation of face recognition [4] with decreased illumination effect can be used.

\section{PROPOSED SYSTEM}

The proposed system is used for tracking govt/private officials by using face recognition and managing the presence of officers in their respective offices. The system architecture is shown in Figure 1. The web cam is interfaced with Raspberry $\mathrm{Pi} 3$ and it is placed where the people enter the office. Camera Module is used to capture the image. From the captured imagethe human faces are processed and extracted by using suitable algorithms. Then detection of face takes place and it automatically verifies with the existing database. 


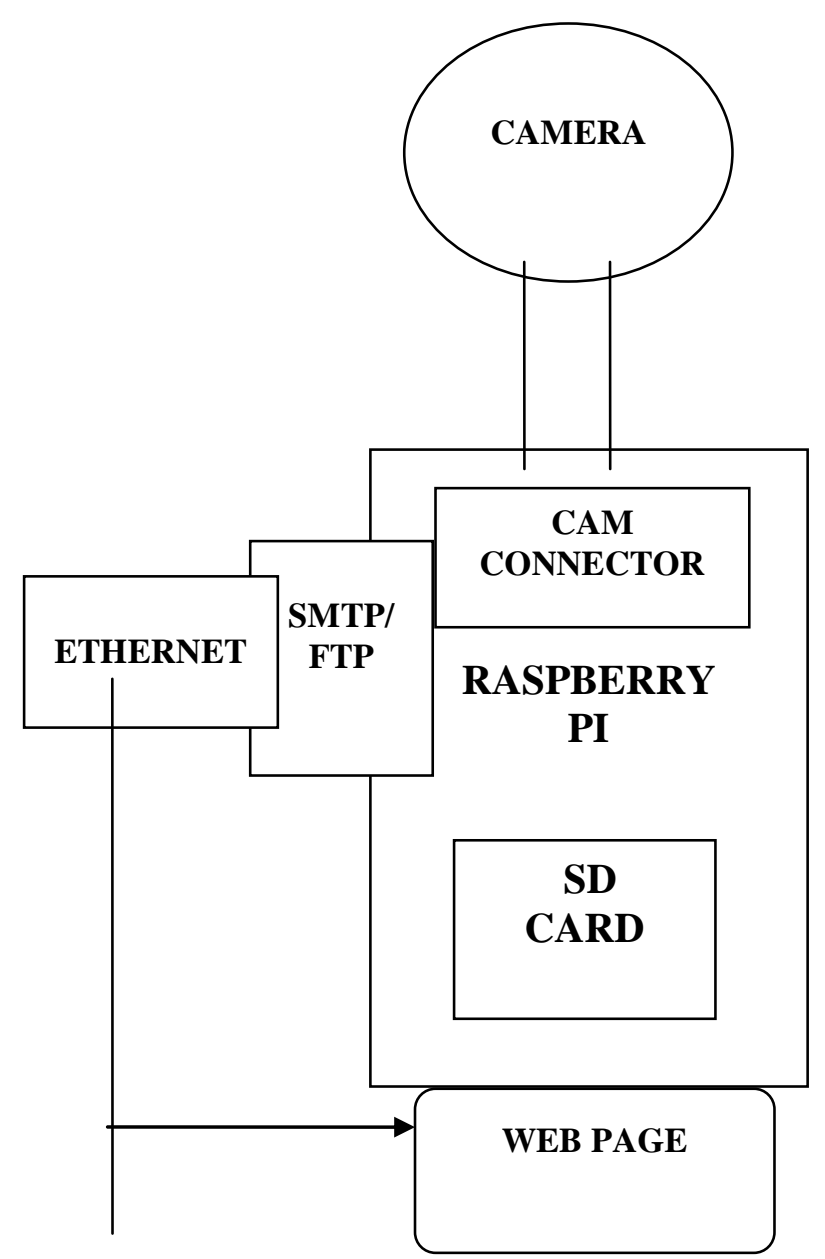

\section{BLOCK DIAGRAM-FIG 1}

Face Recognition is generally more advanced and efficient than other systems. The steps involved are given as follows.

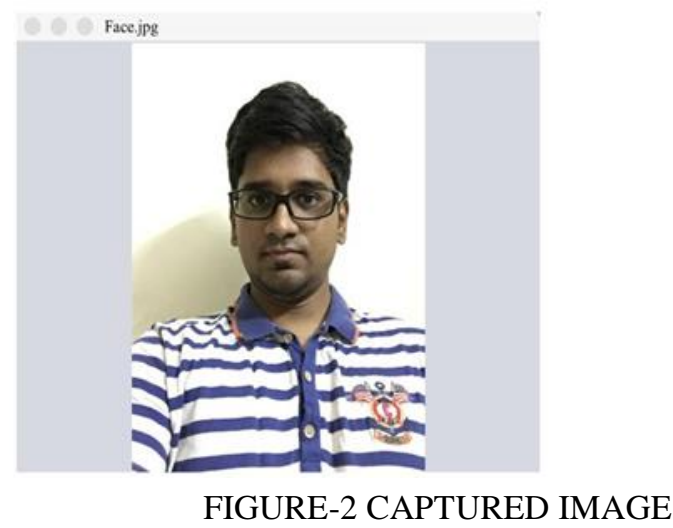

\section{A. Capturing the image}

The camera module is placed in a region where the people enter into organization or office and video is recorded within the distance of less than 5 meters. A camera is used for recording video which contains many frames from which the pixels can be noted and using that pixel's face can be spotted.

\section{B. Creating database}

Currently finger print based biometric attendance is in implementation at most of the offices, it is very difficult for enlistment of every individual whose presence to be tracked. The face of every officers in the government organization should be captured and stored in a suitable database which includes the person's name and their other credentials. Here many numbers of samples are taken for a single individual with different climatic conditions. A group of databases of officers along with more than 8 images of each individual persons is used for recognition.

\section{Detecting Faces}

On preferring an efficient algorithm for recognition of everyone face is critical in this proposed work. Many numbers of algorithm are in use for detectingfaces using OpenCV such as Eigenfaces, Fisher faces and Local Binary Pattern Histograms. Considering the need for the real-time recognition an algorithm which hasbeen opted is the Haar Cascade Algorithm [5] for face detection and recognition. This algorithm is available for use in OpenCV source library [6] and has proved to be robust [7] 


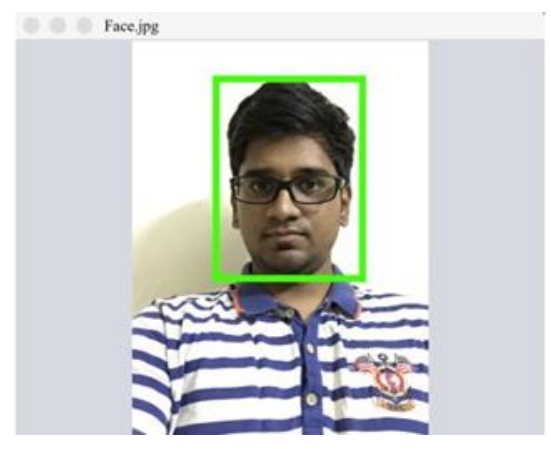

FIGURE-3 DETECTED FACE

\section{Preprocessing}

Since an image may contain unnecessary background noises and elements other than faces it is important to remove those elements. Thus, feature extraction is key for reducing the image to only a face available in the image. Using this method, the size of image is reduced to $150 \times 150$. Histogram equalization is performed on the reduced image and thus the image becomes easier to process.

\section{E. Face Recognition and Classification}

The ability of an algorithm to recognize faces [8] depends upon. Using this algorithm, the feature extraction is done through Principal Component Analysis (PCA). On comparison with many algorithms we have chosen this algorithm for feature extraction and have arrived at this method based on the analysis and results of various methods. Among all those algorithms this one was the first method to represent faces quickly. The values of images are represented as eigenfaces and their corresponding working are used.

An Image in PCA is represented mathematically as,

$$
\chi=A Y+\mu
$$

where $\chi$ is the face vector, $Y$ is the vector of eigenfaces, the average factor and $A$ is the feature vector.

Since faceshave been detected, the next step in this work is to perform face recognition in the detected faces but if detecting faces using normal photo gives low accuracy. Hence this algorithm is used to convert the high dimensionality of twodimensional images into a smaller set of uncorrelated variables. The algorithm finds the direction in which data shows the greatest variance and this direction is called as principal components.

\section{F. POST PROCESSING}

In the work, after the faces are recognized it is stored in a MySQL database and they are updated based on them in and out times. The data present can be accessed by administrator alone.

\begin{tabular}{|l|l|l|l|}
\hline S.NO & Date & Time & Name \\
\hline 1 & $09 / 03 / 2019$ & $9: 00$ & Officer 1 \\
\hline 2 & $09 / 02 / 2019$ & $11: 00$ & Officer 2 \\
\hline
\end{tabular}

Table.1DatabaseSheet

\section{COMPONENTS USED}

- RASPBERRY PI 3

- SD CARD

- CONNECTING CABLE (HDMI TO VGA)

- WEB CAMERA

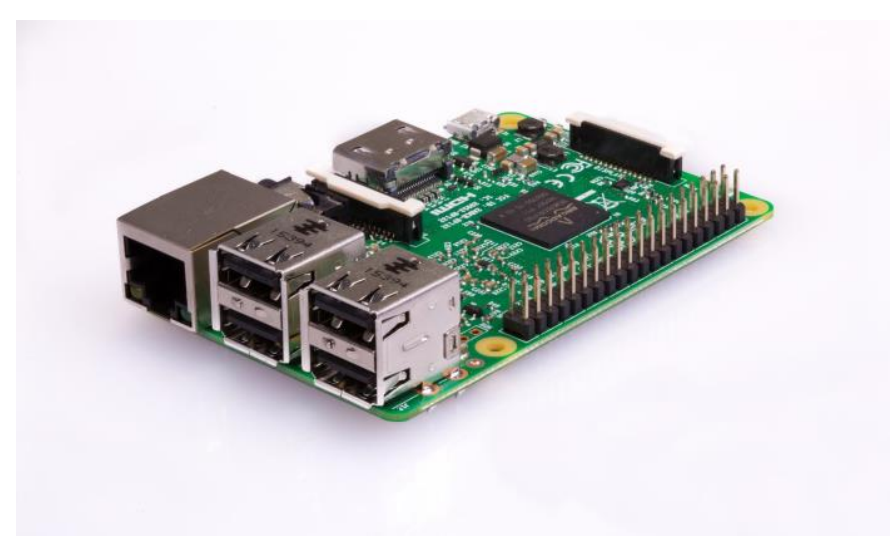

FIG.1RASPBERRY PI 3 MODEL B

\section{PI 3 MODEL B}

5. SPECIFICATIONS:

- Quad Core 1.2GHz Broadcom BCM2837 64bit CPU

- 1GB RAM 
International Journal of Research in Advent Technology, Vol.7, No.3, March 2019 E-ISSN: 2321-9637

Available online at www.ijrat.org

- BCM43438 wireless LAN and Bluetooth Low Energy (BLE) on board

- 100 Base Ethernet

- 40-pin extended GPIO

- 4 USB 2 ports

- 4 Pole stereo output and composite video port

- Full size HDMI

- CSI camera port for connecting a Raspberry Pi camera

- DSI display port for connecting a Raspberry Pi touchscreen display

- Micro SD port for loading your operating system and storing data

- Upgraded switched Micro USB power source up to $2.5 \mathrm{~A}$

- Heater Voltage: 5.0V.

- Dimensions: $18 \mathrm{~mm}$ Diameter, $17 \mathrm{~mm}$ High excluding pins, Pins - 6mm High.

WEB CAMERA:

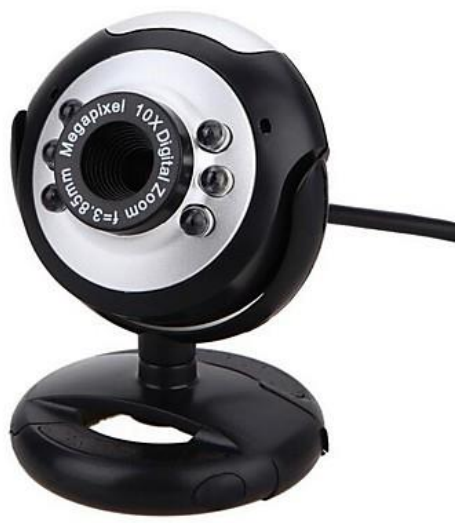

- Connection Type

Corded USB

- USB Type

High Speed USB 2.0

- USB VID_PID

VID_046D\&PID_081A

- Microphone

Built-in, Noise Supression

- Lens and Sensor Type

Plastic

- Focus Type

Fixed

- Field of View (FOV)

$60^{\circ}$

- Focal Length

$4.0 \mathrm{~mm}$

- Optical Resolution
(True)

- Image Capture (4:3 SD)

320x240, 640x480 1.2 MP, 3.0 MP

- Image Capture (16:9 W)

- Video Capture (4:3 SD)

$320 \times 240$, $800 \times 600$

- Video Capture (16:9 W)

360p, 480p, 720p,

- Frame Rate (max)

30fps@640x480

- Video Effects (VFX)

N/A

- Right Light

Right Light 2

- Buttons

Other NA

- Indicator Lights (LED)

- Activity/Power

- Privacy Shade

- $\quad$ No

- Clip Size (max)

- 0 to infinity

- Cable Length

- 5 Feet or 1.5 Meters

\section{WORKING PRINCIPLE}

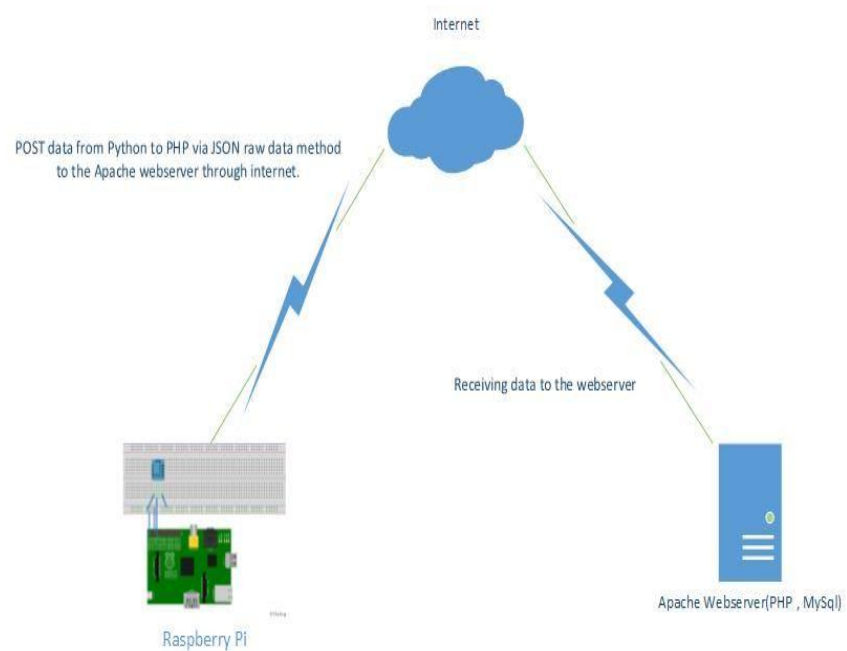

- The Raspberry Pi will get the face detected and upload to webserver. 
- All the data will be POST to the web server via JSON RAW data method.

- Data will store in a data base and publish to a chart.

We can monitor the Temperature and Pollution status with the chart on a webpage

\section{IMPLEMENTATION}

\section{Installing the $\mathrm{OS}$}

When it comes to creating an image on an SD card for your Pi Zero $\mathrm{W}$, there are two options.

When you have to use other operating system instead of Raspbian other options found on NOOBS, that should beinstalled as an own image file on the micro SD card. This idea will be involved only because you need a special *.img file that not only puts your files on the card, but also sets up things like making the card bootable. Raspberry Pi foundation has many more images like Ubuntu, OSMC (Open Source Media Centre), and even Windows 10 IOT Core. You will find out many more if you google it including specialized images for certain tasks. If you've never worked with Raspberry PI before, we recommend Raspbian.

To install your own image on your card, we recommend software called Etcher. This softwarewill provide us all the different steps needed and put them all in one piece of software to take care of everything. Download your image, then run the program, select your image, select your USD card drive, and then hit flash. Once the operating system loaded successfully, remove your card and you are good to go. On completion of installation of image, insert the card into the board and apply power

\section{Software Setup}

hosting a website

\section{step 1: decide the type of website you want}

you will typically find 2 types of websites:

static or basic websites: The websites which are said to be static are simple websites with one or more web pages (called html pages). Also, those web pages can bebuiltby theirown computer with software like dreamweaver and then upload the pages to your host's server using any ftp software (such as filezilla). The changes in the page can be done by editing the pages on your system and then files can be uploaded again. The changes cannot be done dynamically, such websites are called static websites. This type of websitesis cheaper than dynamic websites (below) but come with limited functionalities and no option for e-commerce or interactivity. dynamic websites: These kinds of websites contain information that may vary, depending on the time of day, the viewer and some other factors. The scripts from both clientside and server-side can be used to create and update content. client-side scripts, which run on a user's computer, are mainly used for appearance and interaction purposes. The scripts which are from server-side, which reside on a server and are extensively used by e-commerce and social networking sites, allow users to have individual accounts and provide a customized response for each user. These types of websites are cms-driven, and make you to directly add and edit content (i.e. content, layout, images, and videos), as well as let your visitors leave comments and start discussions. These websites are very suitable for businesses and organizations. The dynamic websites include blogs, forums, photo galleries and e-commerce sites.

\section{step 2: choose your hosting server}

Apart from static html sites which can be hosted on most web servers, when it comes to web applications, there are basically two types of hosting platforms. We can choose depending upon our hosting needs. Here we can explain about the details of some commonly used hosting that is nothing but Linux hosting, which allows running scripts written in php, perl, python and other languages which are originated with-Unix, and usually supports postgresql and mysql databases. this is the most commonly used system today.

In the other hand windows hosting, which allows running asp scripts utilizing .net and other microsoft technologies, and supports microsoft sql server and access database.

\section{step 3: select your web hosting plan}

There are wide range of services in web hosting, such as: shared hosting, in this type of hosting you get to share the physical serverwith other website owners. Even though, you will have your own separate account (secured with login access credentials). This type of hosting is very affordable because the cost for operating the server is shared between you and the other website owners.

vps hosting (virtual private server hosting) In this type, every website is loaded on a very powerful server that is spitted into various number of virtual compartments. The software for the server is configured independently so that each and every unit can function independently. It should be your preferred option for some high-security concerns but don't want to invest in a faster (but costlier) dedicated server.

dedicated hosting: Thishosting offers you complete server of your own, thereby making it faster, more secure... and costlier. it is the great solution for larger businesses and high-traffic websites because it allows maximum number ofcustomizations, configuration, installation and flexibility. 
cloud hosting: This one allows you to have multiple virtual servers (clouds) to work together to host a website or a group of websites It provides countless ability to handle sudden traffic spikes. An Internet-hosted website is not limited to a single server, and the resources allocated to it can shrink or extend dynamically, depending on how much traffic you get It is a major advantage for large websites, including e-commerce websites, newsletters and blogs.

\section{step 4: change your dns address}

After purchasing your web hosting, you will get name servers (also known as domain name servers or dns) - which is the internet's equivalent of a phone book that contains ip addresses $^{3}$.

to get your website up and working, you will need to change the name servers of your domain. It is aeasy but very important step for you to get started.

go to your domain control panel via http://manage.hostgator.in/customer.

enter your registered email address and password.

click on the name of the domain for which you need to change the server name.

in the domain registration section, click on the name server option.

Replace the existing Name Servers with the ones provided by your current web host, and click on the Update Name Servers button.

If the name of the domain is registered with a third-party provider, you will need to log in to their Control Panel, update the Name Servers of the domain to those provided by HostGator. However, if your domain is already using the Name Servers of the third-party provider, you can add an A Record for the domain pointing to HostGator's Server IP in the third-Party DNS Zone. After changing your DNS, it will take about 24-48 hours for your website to start resolving to HostGator India's servers.

\section{Step 5: Upload Your Website}

Now we can upload our website content to our account by connecting to the server using either cPanel's File Manager or FTP Client (such as FileZilla) - after which your website will go live.

Here are the steps to Upload Your Website Using cPanel File Manager

Log in to your cPanel.

Click on the icon titled File Manager.

Select Web Root and click on Go.

All the files and folders should be added under public_html and their respective domain folder.

How to Upload Your Website Using FTP Client
Now we can connect to FTP client via an FTP program such as FileZilla Client. It allows you to see the files and folders on our server like you'd see them on your computer. Now we can use it to drag/drop the website files to the /public_html/ folder.

Steps toconnect web server via FileZilla, follow these steps:

Install FileZilla and open it

From the File menu, select Site Manager

Click on New Site

Name to be given to New Site - such as with your real domain name

The website's IP address should be entered in the field marked asFTP Address

The username and password should be entered which you received through your welcome mail

The port has to be set as 21 (FTP always runs on Port 21)

Click Connect

When our FTP is connected, we can able to see the files and folders of your:

Local computer on the left

Web hosting service on the right

For uploading the files to the hosting service provider via FileZilla, these steps to be carried out:

From the left-hand side of FileZilla, select the file(s) and folder(s) you want to upload.

Drag and drop the file(s) and folder(s) to the directory location on the right side of your web hosting service. FileZilla will now start uploading.

After uploading all the files once, it is finished, FileZilla log will confirm success and your uploads will be visible on the right-hand side.

\section{GRAPHICAL USER INTERFACE(GUI)}

The GUI was developed by using JFrame format present in Java that is installed in Raspberry Pi3. By using Java-SQL connector the database can be easily managed through PhpMyAdmin. There are following functions in the database: Date, Time In, Time Out, Present/Absent, Number of Hours can be added in the future implementation.

\section{SCOPE AND FUTURE WORK}

This module can also be utilized for several security applications where authentication is needed to access the privileges of the respective system. It can also be used to track the peoplewho are all involving in unauthorized business. Face recognition algorithm can be improved with respect to the utilization of resources so that the project can recognize a greater number of faces at a time which can make the system far better. Many numbers of modules can be developed and utilized for home security and personal or organizational benefits. We can also trace a particular higher official in an 
organization quickly and efficiently with the help of this system.

\section{CONCLUSION}

Face recognition attendance systems can thus be proved to be secure and efficient. For real time implementation, the Haar Cascade Classifiers outperforms other algorithms[10] and found to be suitable for implementation of this work. It gives a better recognition rate with a low false rate. The usage of Raspberry Pi independently improves the mobility of the work and it acts as a standalone hardware. This can be further improved by increasing recognition rate[11] and by using Raspberry Pi Infra Red camera module this system can be used as a security surveillance system.

\section{REFERENCES}

[1] G. T.S.Lim Fac., Ayer Keroh,Malaysia S. C. Sim ; M. M. Mansor. 2009.RFID based attendance system Industrial Electronics and Applications. ISIEA 2009. IEEE Symposium on (Vol. 2).

[2] Quan-Xi, Li Gang M. 2012. An Efficient Automatic Attendance System Using Fingerprint Reconstruction Technique IJCSIS International Journal of Computer Science and Information Security.

[3] U. Eze peter; Owerri Nigeria; C.K.A. JoeUzuegbu; Laz Uzoechi; F.K. 2013. Opera biometric based attendance system with remote real time monitoring tertary institutions in developing countries emerging and sustainable technologies for power and ICT in a developing society NIGERCON, IEEE.
[4] Design and implementation of iris based attendance management system Electrical Engineering and Information Communication Technology (ICEEICT), 2015 International Conference.

[5] Adam Schmidt, Andrzej Kasinski, "The Performance of the HaarCascade Classifiers Applied to the Face and Eyes Detection", ComputerRecognition Systems 2016.

[6] Amit Kumar, P.K. Varma, Srinivas Perala, P. R. Chadha, "AutomaticAttendance System By Visual Programming Language LabVIEW," in IEEE International Conference On Power Electronics, Intellegent Control and Energy System 2016

[7] Ruian Liu Mimi Zhang Shengtao Ma, "Design of Face Detection and Tracking System", 2010 IEEE 3rd International Congress on Image and Signal Processing.

[8] Automated attendance management system based on face recognition algorithms Computational Intelligence and Computing Research ICCIC), 2013 IEEE International Conference.

[9] Yung-Mao Lu ; Nat. Kaohsiung Univ. of Appl. Sci., Kaohsiung; BinYih Liao; Jeng-Shyang Pan. 2008. A face recognition algorithm decreasing th illumination effect intelligent information hiding and multimedia signal processing. 2008 IIHSP ${ }^{\text {ee }} 08$ IEEE.

[10] Rafael C. Gonzalez. 2009. Digital Image Processing. Pearson Education India.

[11] Yi-Qing Wang. 2014. An Analysis of the Viola-Jones Face Detection Algorithm. Image Processing On Line. 4: 128-148. 